\title{
Second-Language Learning Ability Revealed by Resting-State Functional Connectivity
}

\author{
Lydia Vinals \\ MRC Cognition and Brain Sciences Unit, Cambridge, CB2 7EF, United Kingdom, and Department of Theoretical and Applied Linguistics, University of \\ Cambridge, Cambridge CB3 9DP, United Kingdom \\ Review of Chai et al.
}

Over the past decade, the cognitive neuroscience of language has gradually shifted its focus from describing the function of specific brain regions to characterizing the spatial and temporal dynamics of functional networks connecting these regions. Examining resting-state functional connectivity (RSFC), the temporal correlation of spontaneous brain activity fluctuations at rest, is a powerful method to characterize neural networks (Fox et al., 2007; Smith et al., 2013). Using this approach, a resting-state network has been shown to be spatially congruent with the language network typically evoked during linguistic tasks (Hampson et al., 2002; Tomasi and Volkow, 2012).

Several studies have shown that RSFC reflects individual differences in performance on various cognitive tasks (Stevens and Spreng, 2014). RSFC strength within the language network has been positively correlated with reading competency (Koyama et al., 2011) and semantic processing ability (Wei et al., 2012). Stronger RSFC within the language network has also been associated with better second-language phonological (Ventura-Campos et al., 2013) and lexical learning (Veroude et al., 2010). Given the striking variability in second-language at-

Received March 19, 2016; revised April 25, 2016; accepted April 27, 2016. The authors declare no competing financial interests.

Correspondence should be addressed to Lydia Vinals, Department of Theoretical and Applied Linguistics, University of Cambridge, 9 West Road, Cambridge, CB3 9DP, United Kingdom. E-mail: Iv288@cam.ac.uk or Lydia.Vinals-Castonguay@mrc-cbu.cam.ac.uk.

DOI:10.1523/JNEUROSCI.0917-16.2016

Copyright $\odot 2016$ the authors $\quad 0270-6474 / 16 / 366141-03 \$ 15.00 / 0$ tainment among adult learners, RSFC measures hold promise for investigating the basis of individual differences in secondlanguage learning. However, previous studies in this area have related pretraining RSFC to learning second-language features trained in the laboratory over relatively short timescales (Veroude et al., 2010; Ventura-Campos et al., 2013). In a recent study in The Journal of Neuroscience, Chai et al. (2016) extend previous work to consider whether pretraining RSFC may account for individual differences in learning outcomes of a second language trained in a naturalistic environment over an extended timescale.

Chai et al. (2016) obtained resting-state data from 15 English native speakers before they began an intensive 12 week French immersion program aimed at developing communication and reading skills through classroom instruction and interactions with native speakers. Pretraining RSFC was then related to improvements in French lexical retrieval and reading ability measured before and after the training program. The authors assessed lexical retrieval by counting the number of words correctly produced by participants when asked to speak in French for 2 min. Reading ability was indexed by calculating the number of words participants read per minute when instructed to read short French paragraphs. Participants improved on both measures upon completing the immersion program.

To estimate RSFC within the language network, Chai et al. (2016) performed a seed-to-voxel analysis on resting-state data by computing temporal correlations between brain activity fluctuations from two a priori seeds, the left anterior insula/ frontal operculum (AI/FO) and the visual word form area (VWFA), and every brain voxel. Contributions of the left AI/FO and VWFA to lexical retrieval and reading, respectively, are suggested in both $\mathrm{fMRI}$ and lesion studies (Price, 2012). The authors found that participants with stronger pretraining RSFC between the left AI/FO and the left posterior superior temporal gyrus (pSTG) as well as between the left AI/FO and the dorsal anterior cingulate cortex (dACC) showed greater improvements in French lexical retrieval. Participants with stronger RSFC between the VWFA and the left mid-STG exhibited greater improvements in French reading ability. The left pSTG and left mid-STG are putative language processing areas; and although the dACC is associated with domaingeneral cognitive control processes, it is a common locus for bilingual language control. These results provide compelling evidence for RSFC strength within the language network reflecting individual differences in second-language learning ability. It should be noted, however, that the study relies on a small sample size and reports very high correlations calling for caution in interpreting the results (Vul et al., 2009). Robust correlation analyses to deal with potential outliers, confirmation of the test-retest reliability of the learning and/or RSFC measures, and replications 
in independent samples would help corroborate the findings.

Chai et al. (2016) suggest that greater RSFC between the left AI/FO and the left pSTG may facilitate phonological and semantic processes supporting second-language lexical retrieval. Although this account is consistent with the established role of these regions in spoken word production, the authors do not explain how these interactions may support improvements in second-language lexical retrieval. One possibility is that interactions between the left AI/FO and the left pSTG support the resolution of lexical competition. Recent studies suggest that lexical retrieval in spoken word production involves the activation of a set of phonologically/semantically related candidate words in left superior and middle temporal regions and competitive selection of the intended word by inferior frontal regions (Abel et al., 2009; Peramunage et al., 2011). Stronger RSFC between the left AI/FO and the left pSTG may facilitate managing the conflict arising when new words in the second language integrate the mental lexicon and compete with words in the first language for selection. This proposal could be substantiated by assessing whether participants with greater pretraining RSFC between these regions also show less disturbance under conditions of increased phonological/semantic competition both between and within language.

Chai et al. (2016) propose that greater pretraining RSFC between the left AI/FO and the dACC may reflect better domaingeneral cognitive control processes contributing to improvements in second-language lexical retrieval. These claims are supported by recent longitudinal functional connectivity studies examining the role of cognitive control in second-language lexical development, which report stronger functional connectivity within the cognitive control network (including dACC) in the early stages of learning (Ghazi Saidi et al., 2013; Grant et al., 2015). These findings are consistent with the convergence hypothesis of bilingual processing (Green et al., 2006), which posits that beginner second-language learners rely on cognitive control processes more than advanced learners to resolve cross-linguistic competition. With increased second-language proficiency, learners become less reliant on cognitive control processes as the first language comes to require less inhibition. On this view, better cognitive control processes are particularly important in the early stages of secondlanguage learning. Better cognitive control processes may also subtend internal speech monitoring. A recent study reports involve- ment of the dACC, bilateral insula and inferior frontal regions in error detection during speech production (Gauvin et al., 2016). A tenable suggestion is that stronger RSFC between the left AI/FO and the dACC may facilitate error monitoring, which may be particularly important in the early stages of second-language learning where lexical retrieval is more demanding and error prone. Therefore, an analysis of the errors produced by participants in the Chai et al. (2016) study and how they relate to these RSFC patterns could prove informative.

Stronger pretraining RSFC between the VWFA and the left mid-STG in participants whose second-language reading ability improved is interpreted by Chai et al. (2016) as reflecting the potentially more efficient mapping of visual input from written words to phonological and semantic representations. This account implies bottom-up processing of orthographic information activating phonological and semantic representations. An alternative account put forward by interactive activation models (e.g., Price and Devlin, 2011) suggests the VWFA integrates bottom-up orthographic information with top-down predictions from phonological and semantic language areas. These top-down predictions play a particularly important role in the early stages of learning because they generate prediction errors, which iteratively work to improve predictions. In addition, evidence for reduced RSFC between left posterior temporal areas (including VWFA) and other regions within the language network in developmental dyslexia has been interpreted as a difficulty in integrating bottom-up sensory inputs with top-down predictions (Schurz et al., 2015). One possibility is that stronger interactions between the VWFA and the left mid-STG may facilitate access to top-down predictions and support second-language reading. An integrative view of the VWFA also raises questions as to whether similarities between the first and the second language may mediate the relationship between these RSFC patterns and second-language reading ability. Better access to top-down predictions may benefit reading in a second language that has strong orthographic overlap with the first language (as French with English) but may not be as helpful for an orthographically distant language for which orthographic information cannot elicit top-down predictions.

RSFC's promise in furthering our understanding of individual differences in second-language learning is not restricted to seed-to-voxel analyses as used by Chai et al. (2016). Further investigations are likely to take advantage of data-driven graph-theo- retical analyses that provide measures of network topology without relying on selecting seeds a priori (Bullmore and Sporns, 2009). Graph theoretical measures allow examining large-scale network-level alterations across the whole brain and provide information regarding network hubs, global and local efficiency, as well as node clustering. Using a graph-theoretical analysis, a recent study investigating individual differences in second-language word-tosound learning in adults found that the degree and local efficiency of the left STG were positively correlated with learning outcomes (Deng et al., 2016). These results suggest that RSFC can account for individual differences in second-language learning success in the absence of a priori hypotheses guiding the analysis. As such, these types of analyses may provide a more objective alternative to investigating how RSFC relates to individual differences in second-language learning ability.

In conclusion, Chai et al. (2016) provide evidence for pretraining RSFC strength accounting for individual differences in second-language learning outcomes in lexical retrieval and reading ability. The authors augment previous findings by considering a second language trained in an intensive, naturalistic environment. Further examination and characterization of RSFC interactions within the language network and how they relate to learning outcomes in a range of linguistic domains will hopefully lead to a more thorough understanding of the mechanisms subtending individual differences in second-language learning. The Chai et al. (2016) findings thus constitute a promising first step in an exciting research endeavor, which will likely capitalize on developments in RSFC analysis techniques.

\section{References}

Abel S, Dressel K, Bitzer R, Kümmerer D, Mader I, Weiller C, Huber W (2009) The separation of processing stages in a lexical interference fMRI-paradigm. Neuroimage 44:1113-1124. CrossRef Medline

Bullmore E, Sporns O (2009) Complex brain networks: graph theoretical analysis of structural and functional systems. Nat Rev Neurosci 10:186-198. CrossRef Medline

Chai XJ, Berken JA, Barbeau EB, Soles J, Callahan M, Chen JK, Klein D (2016) Intrinsic functional connectivity in the adult brain and success in second-language learning. J Neurosci 36:755-761. CrossRef Medline

Deng Z, Chandrasekaran B, Wang S, Wong PC (2016) Resting-state low-frequency fluctuations reflect individual differences in spoken language learning. Cortex 76:63-78. CrossRef Medline

Fox MD, Raichle ME (2007) Spontaneous fluctuations in brain activity observed with func- 
tional magnetic resonance imaging. Nat Rev Neurosci 8:700-711. CrossRef Medline

Gauvin HS, De Baene W, Brass M, Hartsuiker RJ (2016) Conflict monitoring in speech processing: an fMRI study of error detection in speech production and perception. Neuroimage 126:96-105. CrossRef Medline

Ghazi Saidi L, Perlbarg V, Marrelec G, Pélégrini-Issac M, Benali H, Ansaldo AI (2013) Functional connectivity changes in second language vocabulary learning. Brain Lang 124:56-65. CrossRef Medline

Grant AM, Fang SY, Li P (2015) Second language lexical development and cognitive control: a longitudinal fMRI study. Brain Lang 144:35-47. CrossRef Medline

Green DW, Crinion J, Price CJ (2006) Convergence, degeneracy, and control. Lang Learn 56:99-125. CrossRef Medline

Hampson M, Peterson BS, Skudlarski P, Gatenby JC, Gore JC (2002) Detection of functional connectivity using temporal correlations in MR images. Hum Brain Mapp 15:247-262. CrossRef Medline

Koyama MS, Di Martino A, Zuo XN, Kelly C, Mennes M, Jutagir DR, Castellanos FX, Milham MP (2011) Resting-state functional connectivity indexes reading competence in children and adults. J Neurosci 31:8617-8624. CrossRef Medline

Peramunage D, Blumstein SE, Myers EB, Goldrick M, Baese-Berk M (2011) Phonological neighborhood effects in spoken word production: an fMRI study. J Cogn Neurosci 23: 593-603. CrossRef Medline

Price CJ (2012) A review and synthesis of the first 20 years of PET and fMRI studies of heard speech, spoken language and reading. Neuroimage 62:816-847. CrossRef Medline

Price CJ, Devlin JT (2011) The Interactive Account of ventral occipitotemporal contributions to reading. Trends Cogn Sci 15:246-253. CrossRef Medline

Schurz M, Wimmer H, Richlan F, Ludersdorfer P, Klackl J, Kronbichler M (2015) Resting-state and task-based functional brain connectivity in developmental dyslexia. Cereb Cortex 25: 3502-3514. CrossRef Medline

Smith SM, Vidaurre D, Beckmann CF, Glasser MF, Jenkinson M, Miller KL, Nichols TE, Robinson EC, Salimi-Khorshidi G, Woolrich MW, Barch DM, Ugurbil K, Van Essen DC (2013) Functional connectomics from resting-state fMRI. Trends Cogn Neurosci 17: 666-682. CrossRef Medline

Stevens WD, Spreng RN (2014) Resting-state func- tional connectivity MRI reveals active processes central to cognition. Wiley Interdiscip Rev Cogn Sci 5:233-245. CrossRef Medline

Tomasi D, Volkow ND (2012) Resting functional connectivity of language networks: characterization and reproducibility. Mol Psychiatry 17:841-854. CrossRef Medline

Ventura-Campos N, Sanjuán A, González J, Palomar-García MÁ, Rodríguez-Pujadas A, Sebastián-Gallés N, Deco G, Ávila C (2013) Spontaneous brain activity predicts learning ability of foreign sounds. J Neurosci 33:92959305. CrossRef Medline

Veroude K, Norris DG, Shumskaya E, Gullberg M, Indefrey P (2010) Brain and language functional connectivity between brain regions involved in learning words of a new language. Brain Lang 113:21-27. CrossRef Medline

Vul E, Harris C, Winkielman P, Pashler H (2009) Puzzlingly high correlations in fMRI studies of emotion, personality, and social cognition. Psychol Sci 4:274-290. CrossRef Medline

Wei T, Liang X, He Y, Zang Y, Han Z, Caramazza A, Bi Y (2012) Predicting conceptual processing capacity from spontaneous neuronal activity of the left middle temporal gyrus. J Neurosci 32:481-489. CrossRef Medline 